\title{
GANHO DE FORÇA E FUNÇÃO EM IDOSOS POR TREINO ISOMÉTRICO COM E SEM RESPOSTA VISUAL
}

\author{
STRENGTH AND FUNCTION GAINS IN THE ELDERLY FROM ISOMETRIC TRAINING WITH \\ AND WITHOUT VISUAL RESPONSE
}

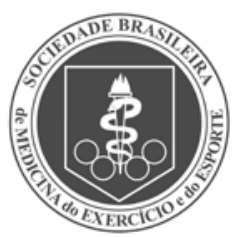

Artigo Original

\author{
AUMENTO DE FUERZA Y FUNCIÓN EN ANCIANOS A TRAVÉS DE ENTRENAMIENTO \\ ISOMÉTRICO CON Y SIN RESPUESTA VISUAL
}

\begin{abstract}
Mauricio Ferraz de Arruda'
(Fisioterapeuta)

Jéssica Aparecida Bazaglia

(Fisioterapeuta)

Gabriela Saravalli' (Fisioterapeuta)

Lucas Langoni Cassettari²

(Fisioterapeuta)

Helena Ribeiro Souza'

(Bióloga)

1. Departamento de Ciências da Saúde do IMES Instituto Municipal de Ensino Superior de Catanduva, SP, Brasil.

2. Departamento de Bases Gerais da Cirurgia da Faculdade de Medicina da Unesp de Botucatu, Botucatu, SP, Brasil.
\end{abstract}

\section{Correspondência:}

Rua José Pelegrine no 733, Eldorado, Bebedouro, SP, Brasil, 14706-110.

zigomaticoah@ ig.com.br

\section{RESUMO}

Introdução: A remediação das alterações fisiológicas que sofrem os idosos é um problema com o qual a saúde pública se defronta com frequência. A premência deste trabalho está em minimizar essa remediação e as alterações que ela propicia no âmbito músculo-esquelético, por meio de um trabalho físico condicionante, que busca aumentar a qualidade de vida dessa população. Objetivo: Avaliar o efeito do treinamento físico sob duas ópticas de comando terapêutico, com e sem resposta visual por unidade de pressão, bem como seus possíveis benefícios quanto ao ganho de torque muscular, inerente à funcionalidade citada no questionário de qualidade de vida SF-36, avaliando assim a influência do treinamento físico. Métodos: A avaliação foi realizada por meio de um questionário de qualidade de vida SF-36 e do teste de levantar e sentar, além da aferição da força por meio do esfigmomanômetro modificado (EM). O individuo foi submetido a duas sessões semanais de exercícios durante um mês, com o protocolo de treinamento que constou de três séries de 10 repetições na cadeira flexora e extensora para ambos os joelhos de maneira isométrica. Os dados foram estatisticamente analisados pela análise de variância e desvio padrão $(p<0,05)$ e correlação de Pearson $(p<0,05)$. Resultados: Notou-se melhora no quadro geral dos idosos, sendo que houve aumento estatisticamente significante no estado geral de saúde, redução da limitação por aspectos físicos, redução da dor e no aumento da capacidade funcional, bem como um incremento da força de flexão e extensão, que teve reflexo positivo no teste funcional. Conclusão: Houve correlação extremamente válida entre o ganho de força em flexão com o teste de sentar e levantar, com o qual, apesar de ganho menor comparado ao ganho de força em extensão, estabeleceu-se correlação com o ganho funcional, independentemente da resposta visual ao exercício.

Palavras-chave: idoso, treinamento de resistência, qualidade de vida, teste de esforço, questionários.

\section{ABSTRACT}

Introduction: The remediation of physiological changes affecting the elderly is a problem frequently faced in public health. The urgency of this work is to minimize this remediation and the changes that it induces at the musculoskeletal level, via work done with physical conditioning, seeking to increase the quality of life of this population. Objective: To evaluate the effect of physical training under two therapeutic commands, with and without visual feedback per pressure unit, as well as possible benefits from increased muscle torque, inherent to the functionality mentioned in the SF-36 questionnaire, thus evaluating the influence of physical training. Methods: The evaluation was conducted using an SF-36 quality of life questionnaire and the sit-to-stand exercise, in addition to measurement of strength by means of the modified sphygmomanometer (MS). The subjects underwent two weekly exercise sessions for a month, with a training protocol that consisted of three sets of 10 isometric repetitions on the flexion-extension chair. Data were statistically analyzed by ANOVA and standard deviation ( $p<0.05)$, and Pearson's correlation $(p<0.05)$. Results: We observed an improvement in the general conditions of the elderly, with a statistically significant increase in general health, reduced limitation caused by physical problems, reduced pain and increased functional capacity, as well as an increase in the strength of flexion and extension, which had a positive impact on functional testing. Conclusion: There was highly valid correlation between the strength gained in flexion in the sit-to-stand exercise, which, although lower than the gain in strength in extension, enabled the establishment of a correlation with functional gain, regardless of the visual feedback.

Keywords: elderly, resistance training, quality of life, exercise test, questionnaires.

\section{RESUMEN}

Introducción: La remediación de las alteraciones fisiológicas que sufren los ancianos es un problema con el cual la salud pública se enfrenta con frecuencia. El apremio de este trabajo es minimizar esa remediación y las alteraciones que propicia en el ámbito músculo-esquelético, por medio de un trabajo físico condicionante, que busca aumentar la calidad de vida de esa población. Objetivo: Evaluar el efecto del entrenamiento físico bajo dos ópticas de comando terapéutico, con y sin respuesta visual por unidad de presión, bien como sus posibles beneficios cuanto al aumento 
de torque muscular, inherente a la funcionalidad citada en el cuestionario de calidad de vida SF-36, evaluando asi la influencia del entrenamiento físico. Métodos: La evaluación fue realizada por medio de un cuestionario de calidad de vida SF-36 y del test de levantar y sentar, además de la medición de la fuerza por medio del esfigmomanómetro modificado (EM). El individuo fue sometido a dos sesiones semanales de ejercicios durante un mes, con el protocolo de entrenamiento que fue de tres series de 10 repeticiones en la silla flexora y extensora para ambas rodillas de manera isométrica. Los datos fueron estadísticamente analizados por el Análisis de Variancia y Desvío Estándar $(p<0,05)$ y correlación de Pearson $(p<0,05)$. Resultados: Se notó una mejora en el cuadro general de los ancianos, siendo que hubo mejora estadísticamente significativa del estado general de salud, de la limitación por aspectos físicos, de la reducción del dolor y de la capacidad funcional, bien como un incremento de la fuerza para flexión y extensión que tuvo reflejo positivo en el test funcional. Conclusión: Hubo correlación extremadamente válida entre el aumento de fuerza en flexión con el test de sentarse y levantarse, en el cual, a pesar de aumento menor comparado al aumento de fuerza en extensión, se estableció correlación con el aumento funcional, independiente de la respuesta visual al ejercicio.

Palabras clave: anciano, entrenamiento de resistencia, calidad de vida, test de esfuerzo, cuestionarios.

\section{INTRODUÇÃO}

O envelhecimento é um processo natural presente em todas as espécies, este, na espécie humana, ocorre acompanhado de algumas perdas, como cognitivas, sociais, motoras e metabólicas que levam à diminuição da qualidade de vida desses idosos

Dentre essas perdas, a que mais afeta a funcionalidade, agravando o dia a dia do idoso, é a neuromotora, que em resposta ocorre à diminuição das fibras musculares, em conseqüência a diminuição da força muscular, por isso, a importância do treinamento de força para compensar esta perda².

A atividade física contribui diretamente para a melhora e manutenção das funções do aparelho cardiovascular e locomotor, diminui os efeitos do desuso das doenças crônicas e assim prevenido perdas e incapacidades².

Na vanguarda, a condição de saúde física do idoso vem sendo analisado com maior ênfase, em sua funcionalidade global. Diante desse novo padrão é fundamental analisar a capacidade funcional dessa população: flexibilidade e força dos MMII (membros inferiores) e MMSS (membros superiores), equilíbrio, agilidade e resistência aeróbica. Nesse sentido para a manutenção da capacidade física relacionado aos MMII e conseqüente a capacidade funcional, a participação desses idosos em programas de atividades físicas regulares, torna-se, um caminho efetivo, além de obter um melhor custo benefício. Idosos ativos têm cerca de $30 \%$ a menos de chance de serem acometidos por limitações funcionais moderadas ou severas ${ }^{3}$.

Para alguns autores, a diminuição da força dos membros inferiores no envelhecimento é considerada como relacionada à redução, durante esse período, de atividades físicas como pular e correr; enquanto isso os músculos das mãos são considerados menos propensos a sofrer atrofia e são os que mantêm a sua força com o avançar do envelhecimento, desde que o uso da mão permaneça com uma freqüência importante durante todo esse período ${ }^{4}$.

Foi constatado que em um grupo de indivíduos sedentários com idade de 55 a 70, comparado com um grupo controle, que a realização de exercício físico desencadeou um aumento da atividade metabólica cerebral e a melhoria da função cognitiva. Este autor conclui que os praticantes de programas de exercícios em qual relatam maior sensação de bem estar em relação aos não praticantes ${ }^{5}$.

Os músculos da coxa especificamente apresentam diminuição significativa de sua secção transversa nas pessoas idosas, em comparação com os indivíduos jovens, enquanto os músculos dos membros superiores se atrofiam em menor grau. Sugere-se que esta atrofia dos músculos dos membros inferiores ocorra em função de uma falta de uso, ou seja, que os adultos de idade avançada tem uma tendência de não treinar esses músculos com frequência ${ }^{6}$.

Para isso de exercício devem ser constituídos por atividades de endurance, de flexibilidade e de força dentro da capacidade da população de idosos que esta sendo servida, com o objetivo de obter melhorias nesses componentes do condicionamento físico?.

A atividade física na terceira idade é importante, pois cria um clima descontraído, mobiliza as articulações e aumenta o tônus muscular, proporcionando maior disposição para o cotidiano, buscando objetivos quanto à qualidade de vida como, por exemplo, o bem estar físico, autoconfiança, segurança no dia a dia através do domínio do corpo, elasticidade, melhoria da resistência, auxílio no tratamento contra depressões, decepções e vazios anteriores entre muitos outros benefícios ${ }^{8}$.

Um método alternativo para a mensuração da força muscular no contexto clínico é o teste do esfigmomanômetro modificado (TEM), uma vez que reúne as vantagens do teste muscular manual (TMM) e do dinamômetro portátil, sem apresentar as suas principais desvantagens. O TEM envolve a utilização do esfigmomanômetro aneróide, um equipamento de baixo custo, portátil, facilmente encontrado e comumente adquirido pelos profissionais da área da saúde para mensuração da pressão arterial. Além disso, o TEM é de rápida e fácil execução, segue procedimentos similares aos adotados com o TMM ou o dinamômetro portátil e fornece valores objetivos que podem ser associados a medidas de força muscular?.

A funcionalidade no idoso pode ser analisada através de inúmeros testes, dentre eles o de sentar-levantar (TSL).

O movimento de sentar e levantar de uma cadeira é um dos movimentos mais comuns do dia a dia do ser humano. Desta maneira a postura sentada pode se tornar difícil para os idosos, visto que a mudança desta postura para uma postura ereta exige deslocamento do centro de gravidade de uma postura estável para uma menos estável, através do movimento de extensão das extremidades inferiores exigindo coordenação de todo o corpo e, por tanto, em idosos que possuem limitações de movimento há alterações na cinética do levantar e sentar ${ }^{10}$

Estes movimentos requerem força e coordenação suficientes para proporcionar velocidade adequada na parte superior do corpo, gerando movimento. Para reduzir a progressão anterior do corpo o indivíduo deve ser capaz de utilizar as contrações excêntricas para controlar os músculos do tronco e quadril, evitando deste modo a queda para frente na fase do deslocamento- transferência, a integridade das articulações dos membros inferiores e a força devem estar adequadas para a fase de extensão do levantar, requer um bom controle de contração concêntrica ${ }^{10}$. 
Por exemplo, a capacidade de mudar da posição sentada para em pé é comprometida em torno dos cinqüenta anos e, por volta dos oitenta, essa tarefa se torna impossível para algumas pessoas ${ }^{10}$.

Todos os tipos de avaliação sobre a condição física de um indivíduo não carregam importância se uma conexão com a qualidade de vida não estiver estabelecida na avaliação clínica.

O Medical Outcomes Study 36-Item Short-Form Health Survey (SF-36) é um questionário genérico que avalia aspectos da qualidade de vida que estão diretamente relacionadas à saúde do indivíduo. As limitações físicas têm repercussão sobre o campo emocional de qualquer pessoa. Assim, a depressão e a presença de distúrbios de comportamento são fatores que apresentam grande influência sobre a qualidade de vida do paciente. Neste ponto, o SF-36 oferece uma valiosa ajuda complementando a avaliação física do doente. O SF-36 avalia oito conceitos (ou dimensões) de saúde: capacidade funcional, aspecto físico, dor, estado geral de saúde, vitalidade, aspectos sociais, aspectos emocionais e saúde mental ${ }^{11}$.

O presente trabalho propõe apresentar de forma não inovadora, mas pouco usada, a interferência da mecanoterapia por meio da isometria com meio positivo direcionado a saúde da população geriátrica relevando para melhor obtenção de um ganho de qualidade de vida, propondo-se a mostrar, estabelecer e notificar possíveis benefícios, por meio do questionário de qualidade de vida SF-36, e o teste de sentar e levantar com preceito de treinamento e avaliação. Com este estudo visa-se com a avaliação pressórica do esfignomanômetro, o condicionamento físico por meio da mecanoterapia, e sua aplicação pratica e o real beneficio da resposta visual pressórica de um grupo em detrimento ao outro.

Tendo nestes métodos e terapias um meio de fácil acesso e de grande valia para a sociedade, haja vista que a saúde passa por um processo de descrédito frente a um plano social.

O objetivo deste estudo foi avaliar os efeitos do treinamento físico isométrico resistido na população geriátrica por meio do questionário de qualidade de vida SF-36, teste funcional de levantar e sentar tendo por objetivo avaliar a força dos membros inferiores utilizando para esse treinamento a resposta visual pressórica e em um dos dois grupos distintos também o visual.

\section{CASUÍSTICA E MÉTODOS}

Participaram deste estudo 22 indivíduos de ambos os sexos, com idade de 65 a 75 anos divididos em grupo com resposta visual pressórica (n-11) e sem resposta visual pressórica (n-11), de forma randomizada. Todos os indivíduos ou seus responsáveis assinaram um termo de consentimento, e a pesquisa foi conduzida de acordo com o Conselho Nacional de Saúde, resolução 196-96. E obteve aprovação pelo Comitê de Ética Responsável com parecer n 415.463.

Os critérios de inclusão: foram selecionados 22 indivíduos, maiores de 65 anos, residentes na comunidade, sem restrição de raça e/ou classe social.

Critérios de exclusão: idosos submetidos previamente a cirurgias ortopédicas dos membros inferiores e/ou com história de fratura; aquelas que não foram capazes de deambular sem auxílio e as portadoras de doenças neurológicas. Excluíram-se também idosos que informaram estar com algum tipo de processo inflamatório musculoesquelético na fase aguda, que pudesse interferir nos testes e/ou no programa de treinamento; as que realizavam atividade física de forma regular, duas ou mais vezes na semana; aquelas que informaram neoplasia em atividade nos últimos cinco anos e as que usavam medicamento com ação ampla sobre o sistema imunológico.

\section{MATERIAIS}

Foi utilizado um aparelho mecanoterápico confeccionado exclusivamente para tal, sendo uma cadeira fixa a 90 graus, com $70 \mathrm{~cm}$ chão-assento, $40 \mathrm{~cm}$ borda anterior - borda posterior, $40 \mathrm{~cm}$ encosto toracolombar, $60 \mathrm{~cm}$ anteparo de madeira anterior, $60 \mathrm{~cm}$ anteparo de madeira posterior (figura1), Esfignomanômetro e a versão brasileira do questionário genérico de qualidade de vida SF-36.

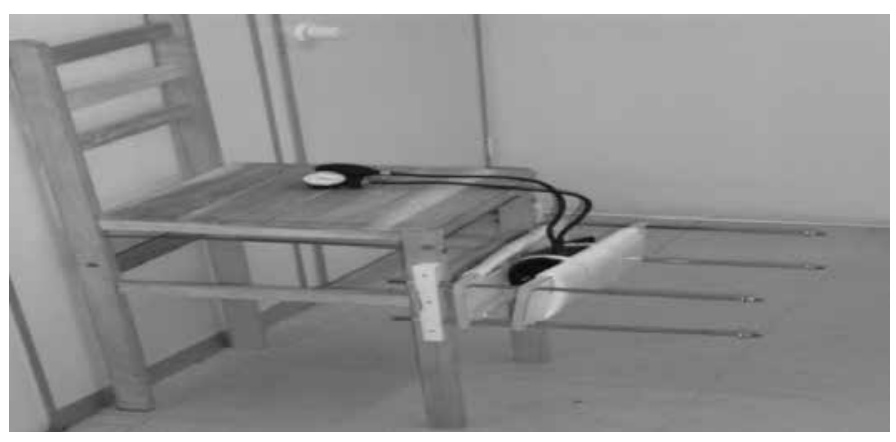

Figura 1. Cadeira confeccionada para atividades isométricas de avaliação de força e realização de treino.

\section{Procedimentos}

Foi aplicado o questionário SF-36, ao individuo de maneira clara, de fácil compreensão, orientado de que a pesquisa questionaria sobre sua saúde e de quão bem e capaz de fazer suas atividades de vida diária.

Foi aplicado o teste levantar e sentar, para avaliação de força e a resistência dos membros inferiores, o qual deverá ser executado em tempo de 30 segundos. Utilizando para o mesmo os seguintes instrumentos - cronômetro, cadeira com encosto e sem braços, com altura de assento de aproximadamente $43 \mathrm{~cm}$. O teste foi realizado da seguinte maneira:

Posição do avaliado - sentado na cadeira com as costas encostadas no encosto e pés apoiados ao chão.

Posição do avaliador - próximo ao avaliado assegurando o bom funcionamento da cadeira e a segurança do participante.

O participante cruza os braços com o dedo médio em direção ao acrômio. Ao sinal o participante ergue-se e fica totalmente em pé e então retorna a posição sentada. O participante e encorajado a completar tantas ações de ficar totalmente em pé e sentar quanto possível em 30 segundos. $\mathrm{O}$ analisador deverá realizar uma vez para demonstrar o teste para que o participante tenha uma aprendizagem apropriada. O teste deverá ser realizado uma vez.

A pontuação foi obtida pelo número total de execuções corretas num intervalo de 30 segundos. Por razões de segurança a cadeira foi colocada contra uma parede de forma a evitar que se mova durante o teste. A aferição da força flexora e extensora foi mensurada através do TEM da seguinte maneira: O esfignomanômetro sempre pré insuflado a $20 \mathrm{mmHg}$ de forma 9 .

Posição do avaliado: Flexão - sentado na cadeira alinhado biomecanicamente com joelho a $90^{\circ}$ realizando contração isométrica para flexão de joelho, (3 repetições mantendo por 5 segundos cada).

Extensão - sentado na cadeira alinhado biomecanicamente realizando contração isométrica para extensão de joelho a 90\% , (3 repetições mantendo por 5 segundos cada) (figura 2).

Descartou-se o melhor e o pior resultado, restando assim à medida alvo.

Posição do avaliador: Próximo à cadeira para orientar a postura, estimular verbalmente o paciente.

Os participantes foram divididos em dois grupos onde um grupo recebeu a resposta visual através da observação do manômetro e o outro somente estimulo verbal.

Após a primeira aplicação do questionário, o individuo foi submetido a duas sessões semanais por 1 mês de exercícios para o fortalecimento do grupamento extensor e flexor do joelho. O protocolo 

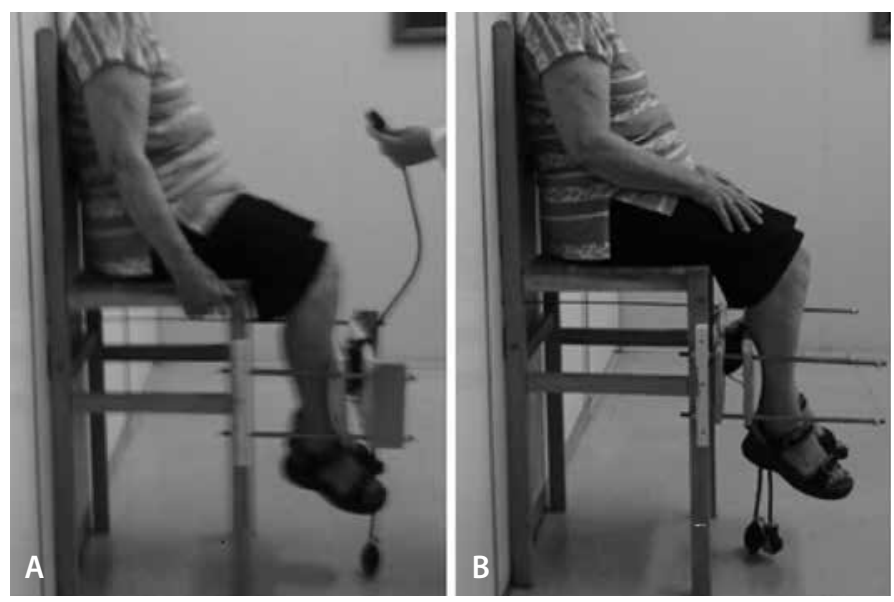

Figura 2. A) Treino isométrico para extensores de joelho; B) Treino isométrico para flexores de joelho com resposta visual e sem resposta visual respectivamente.

de treinamento constou de 3 séries de 10 repetições, por 5 segundos em contração isométrica ${ }^{9}$ (figura 3).

Procedimentos finais: as reaplicações dos testes e do questionário foram feitas seguindo os padrões dos procedimentos iniciais.

\section{Análises estatísticas}

O cálculo amostral foi realizado tendo-se considerado um intervalo de confiança de $95 \%$, um erro de 20\%, um tamanho de efeito de 0,50 e o valor de a de 0,05. Utilizando-se a medida de desempenho funcional, esse cálculo mostrou a necessidade de 11 voluntárias em cada grupo. O teste de LEVENE, foi aplicado a fim de verificar a variância intra- grupos. Para todos os grupos os testes de nível de significância foram de $p<0,05$ (ANOVA, Bonferroni) média e desvio padrão para as variáveis dependentes de antes e depois. O teste de correlação de Pearson foi usado para comparar as variáveis distintas

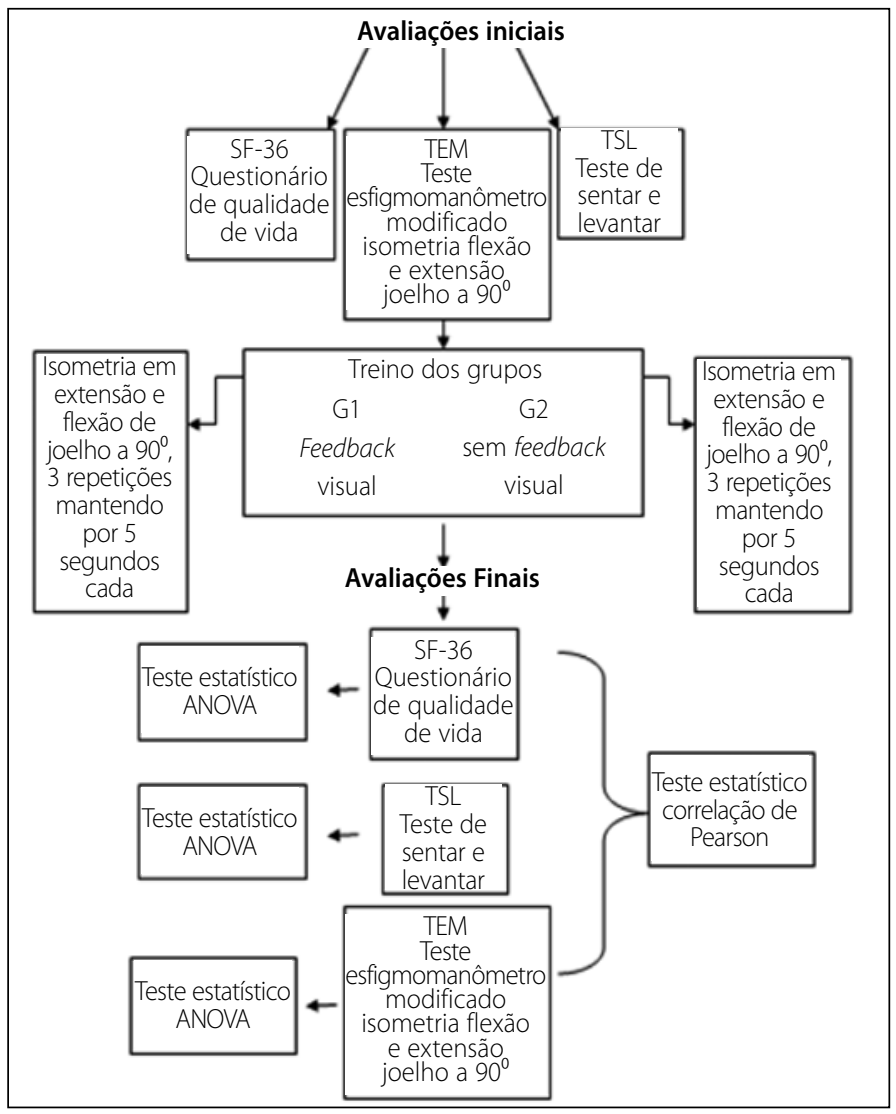

Figura 3. Fluxograma da pesquisa.

\section{RESULTADOS}

Os resultados do presente estudo demonstram de maneira global um ganho consistente na maioria das variáveis relacionadas aos domínios referentes à qualidade de vida bem como quanto ao teste funcional e o ganho de força isométrica para extensão e flexão de joelho. A capacidade funcional referente à avaliação da qualidade de vida se mostrou aumentada para os indivíduos no pós treino em ambos os grupos com e sem resposta visual.

Relativo a limitações por aspectos físicos, demonstrou-se o ganho pós treino em ambos os grupos com e sem resposta visual. O domínio dor demonstra a redução do relato da sintomatologia dolorosa evidente no grupo fortalecido sem resposta visual de acordo com o desvio padrão (DP). De acordo com o estado geral da saúde nota-se o ganho pós- treino em ambos os grupos com e sem resposta visual. A figura 4 expõe as diferenças dos dados interpretados.

Em relação ao teste funcional nota-se um incremento positivo para a ação cotidiana de sentar e levantar tanto para o grupo sem resposta visual quanto para o grupo com resposta visual (figura 5).

De acordo com os resultados obtidos com treino de fortalecimento isométrico para flexão e extensão o ganho foi menor e maior respectivamente (figura 6), o que não indica uma resposta de ineficácia secundária funcional quanto ao pouco ganho de flexão.

\section{A \\ Limitações por aspectos físicos}

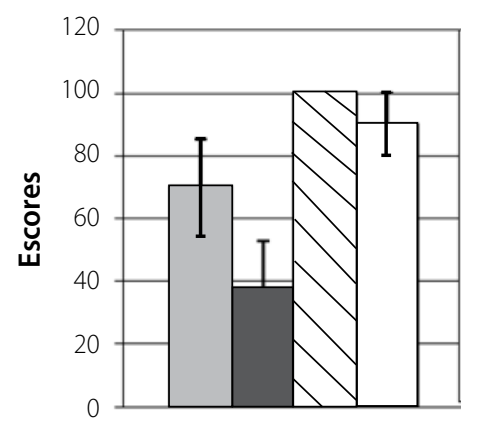

$\square \quad$ Antes s/ resposta visual

- Antes c/ resposta visual

$\Delta \quad$ Depois $s /$ resposta visual

$\square$ Depois C/ resposta visual
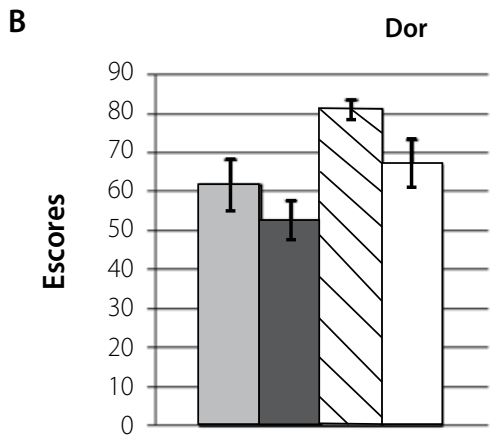

$\square \quad$ Antes s/ resposta visual

$\square \quad$ Antes c/ resposta visual

$\Delta \quad$ Depois s/ resposta visual

$\square \quad$ Depois c/ resposta visual

C Estado geral da saúde

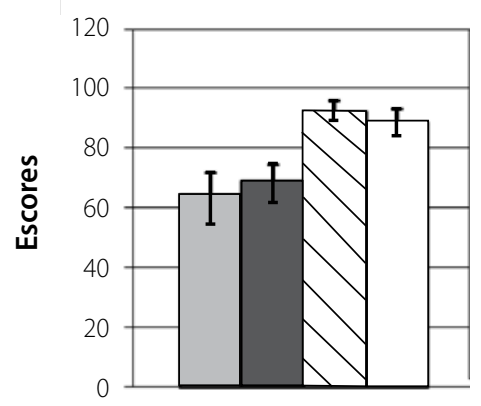

$\square$ Antes s/ resposta visual

- Antes C/ resposta visual

$\nabla \quad$ Depois $s /$ resposta visual

$\square$ Depois Cl resposta visual

Figura 4. Resultados dos domínios referentes à qualidade de vida, demonstrando o antes e depois do treino isométrico com e sem auxílio de resposta visual. 


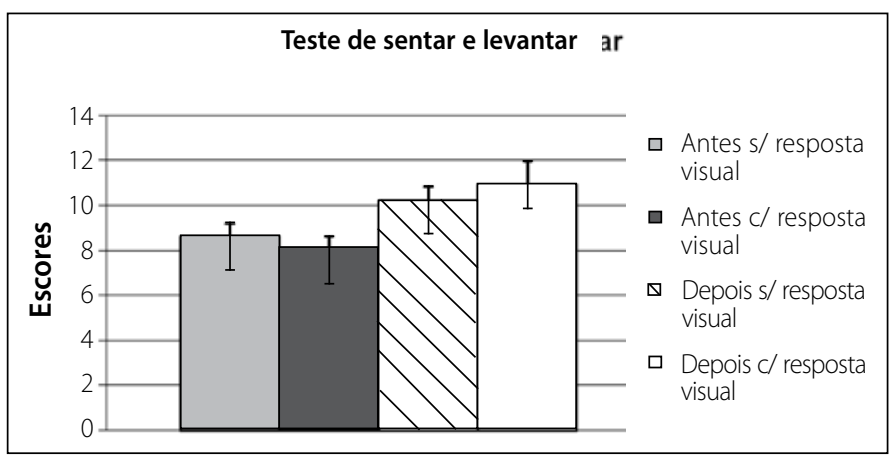

Figura 5. Relativa ao teste de sentar e levantar, que demonstra o ganho pós- treino em ambos os grupos com e sem resposta visual.

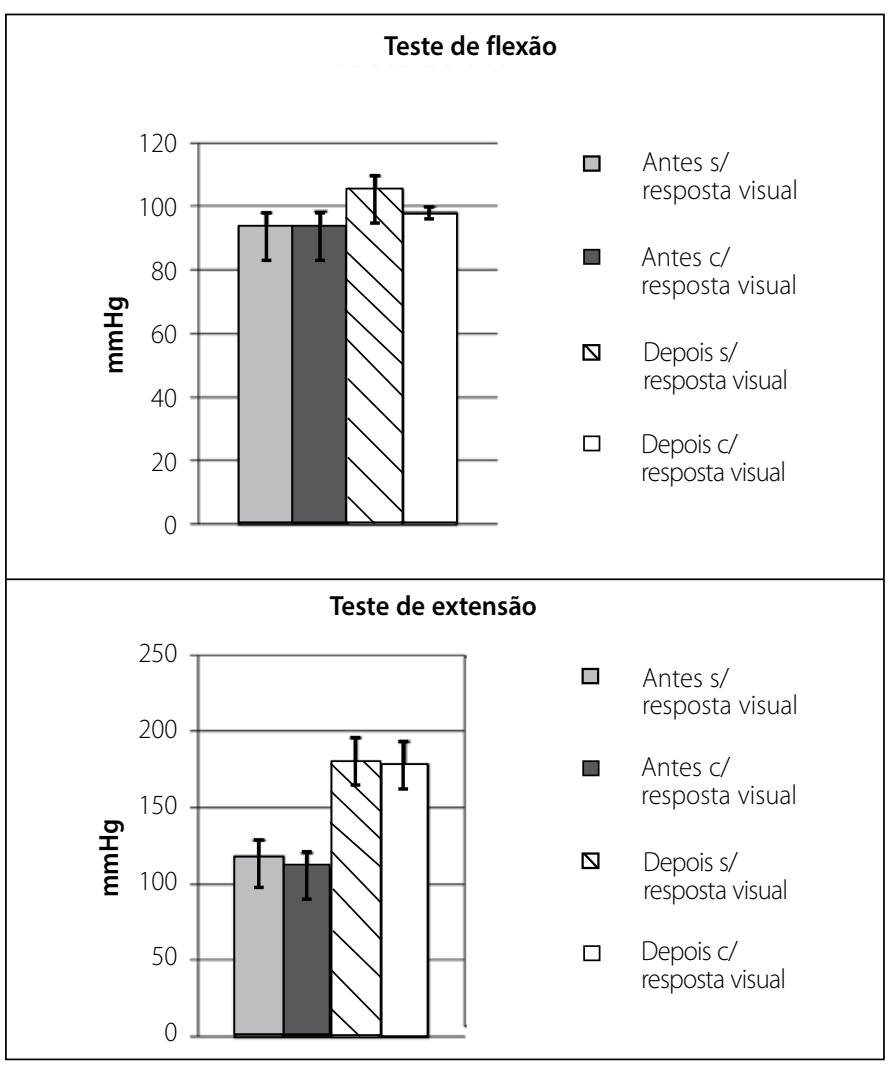

Figura 6. Relativa ao ganho de força em flexão e extensão, que demonstra o ganho pós- treino nos grupos fortalecidos com e sem resposta visual.

Com a aplicação de uma tabela de sétupla entrada em teste de correlação de Pearson, foi gerado correlações entre as variáveis independentes do grupo sem feedback visual, notando-se correlação na intersecção entre o teste de sentar e levantar e a capacidade funcional $p<0,02$, sendo estatisticamente significante com $p<0,005$.

Com a aplicação uma tabela de sétupla entrada em teste de correlação de Pearson, gerou-se correlações entre as variáveis independentes, nota-se correlações nas intersecções entre (capacidade funcional x limitações por aspectos físicos $p<0,0018$, capacidade funcional $x$ dor $p<0,0039$, capacidade funcional $x$ estado geral da saúde $p<0,0021$ ); (limitações por aspectos físicos $x$ dor $p<0,0022$ limitações por aspectos físicos $x$ estado geral da saúde $p<0,0002$ ); (dor $x$ estado geral da saúde $p<0,001$ ); (força de flexão $x$ teste de sentar e levantar $p<0,0478$ ); no grupo com feedback, sendo estatisticamente significante com $p<0,005$.

\section{DISCUSSÃO}

Este trabalho buscou verificar o efeito de um programa de fortalecimento muscular isométrico em cadeira confeccionada especialmente para tal, este fortalecimento foi realizado com a interposição de mecanismo insuflável conhecido como TEM, durante 1 mês o treino foi realizado e a capacidade funcional bem como a força muscular dos extensores e flexores do joelho, e a qualidade de vida dos avaliados.

Os resultados demonstraram que, após a realização do programa, houve melhora quanto ao ganho na força muscular e do desempenho funcional. A literatura atual apresenta uma grande discussão em relação aos programas de fortalecimento muscular propostos para idosos, indicando uma variabilidade no volume, intensidade e duração dos treinamentos de força muscular propostos.

Programas baseados em contrações musculares de baixa velocidade tendem a um maior recrutamento das unidades motoras, contribuindo para um maior desempenho muscular, mas também para um maior acúmulo de metabólitos 3 . Por outro lado, o ganho em potência muscular vem sendo associado a uma maior capacidade de realizar atividades funcionais, melhora da independência e da qualidade de vida dos idosos.

Provavelmente, isso deve ser explicado pelo fenômeno da diminuição das fibras do tipo II nos idosos e suas conseqüências. No entanto, esses estudos encontrados na literatura devem ser comparados ao presente estudo com cautela, pois foram realizados em idosos, mas sem a caracterização da síndrome de fragilidade ${ }^{3}$.

No nosso conhecimento, até o momento, este é o primeiro estudo que realizou um programa de fortalecimento muscular, com percentual de resistência máxima, com a confecção de uma cadeira para tal com o TEM analisando a importância de uma resposta visual no ganho de força e sua resposta secundária funcional.

Nos MMII, os grupos musculares mais frequentemente avaliados e referendados com o TEM foram os extensores do joelho e da articulação coxofemoral. A força dos músculos abdutores da articulação coxofemoral, flexores de joelho, flexores plantares e dorsiflexores do tornozelo foi pouco investigada; já a força de adutores, rotadores internos e externos da articulação coxofemoral não foi avaliada com o TEM. As medidas de força desses músculos têm sido relacionadas à independência na realização de importantes atividades funcionais

Algumas propriedades de medida, como a validade e a confiabilidade, já foram investigadas para o TEM, tendo seus resultados sido reportados na literatura. A avaliação da força muscular isométrica encontra-se como recurso dentre os recursos de avaliação de força como meio fácil mais seguro, confiável e prático de tal maneira que os testes com o TEM tem sido publicados não havendo restrição quanto ao seu uso?.

O programa de fortalecimento utilizado neste estudo não teve características especificas (alta ou baixa velocidade de treino), visando ganho isolado de força ou de potência muscular. O objetivo maior foi testar um programa de exercícios, similar àqueles usados na prática clínica diária, em uma população específica de idosos.

Em nosso estudo todas as variáveis de do questionário de qualidade de vida obtiveram índices melhorados após o treino de força isométrica, bem como a melhora no índice funcional de sentar e levantar, quando comparados os grupos com e sem resposta visual o que nota-se é um aumento equitativo das capacidades sem discrepância em os grupos resposta visual e sem resposta visual, sugerindo uma não importância quanto ao estímulo visual, isso possivelmente deve-se aos dois grupos contarem com comando verbal do examinador durante o exercício.

Fato extremamente importante do trabalho foi o fator correlacional no grupo sem resposta visual entre as sete variáveis analisadas, que em seu resultado final demonstra uma correlação positiva entre o teste de sentar e levantar e a capacidade funcional, ou seja, quanto mais aumenta ou reduza um índice o outro acompanha.

Desta maneira uma análise do grupo com resposta visual tam- 
bém foi feita verificando da mesma forma as sete variáveis de modo a correlacioná-las. Demonstrando uma correlação de real importância que foi entre a força de flexão e o teste de sentar e levantar, ou seja, quanto mais aumenta ou reduza um índice o outro acompanha.

Isso se torna adjuvante a idéia de que o fortalecimento quadricipital é sempre o indicado, mas que há sim a necessidade de fortalecer os flexores de joelho, já que são músculos que atual sinergicamente em cadeia cinética fechada para o membro inferior, visto no presente trabalho esta real correlação apesar de a força quadricipital dos extensores terem tido um evidente e maior ganho em média, foi a força em flexão apesar de um ganho menor estabeleceu-se correlação com o ganho funcional.

Lustosa et al. ${ }^{3}$ compararam ganhos de potência e torque após programas de alta e baixa velocidade. Eles demonstraram que os maiores aumentos de potência ocorreram na sétima e oitava semanas de treino, enquanto o ganho de força foi mais bem observado na terceira e quarta semanas do treinamento

Remontando os resultados para o presente trabalho a potencia teve caráter demarcado indiretamente pela avaliação do teste funcional onde a explosão muscular para esta tarefa mimetiza essa condição, obtendo ganhos expressivos em ambos os grupos.

Apesar de ser esta uma suposição, deve-se lembrar que, em idosos, o maior déficit muscular ocorre nas fibras do tipo $\|^{3}$. Dessa forma, com base nos resultados obtidos, pode-se afirmar que programas que promovam o ganho de potência muscular devem ser considerados para idosos no intuito de melhorar também o seu desempenho funcional.

A literatura atual apresenta uma grande discussão em relação aos programas de fortalecimento muscular propostos para idosos, indicando uma variabilidade no volume, intensidade e duração dos treinamentos de força muscular propostos. Segundo Lustosa et al. ${ }^{3}$, programas baseados em contrações musculares de baixa velocidade tendem a um maior recrutamento das unidades motoras, contribuindo para um maior desempenho muscular, mas também para um maior acúmulo de metabólitos.

Por outro lado, o ganho em potência muscular vem sendo associado a uma maior capacidade de realizar atividades funcionais, melhora da independência e da qualidade de vida dos idosos. Provavelmente, isso deve ser explicado pelo fenômeno da diminuição das fibras do tipo II nos idosos e suas conseqüências. No entanto, esses estudos encontrados na literatura devem ser comparados ao presente estudo com cautela, pois foram realizados em idosos, mas sem a caracterização da síndrome de fragilidade.

Da mesma forma, a utilização de cargas elevadas pode contribuir para um maior acúmulo de metabólitos e um esgotamento dos substratos de energia. Neste estudo, foi determinado a isometria, o número de séries (três para cada exercício) e o número de repetições. No entanto, houve preocupação com o tempo de manutenção da contração, obedecendo a critérios da literatura referendada?

Assim a perda de potência pode ser mais danosa ao idoso do que a perda de força muscular máxima, pois o desenvolvimento de força explosiva é um importante mecanismo na prevenção de quedas e na realização de tarefas pesadas, tais como rapidez para ficar em pé e caminhar.

Apesar de ter sido conduzido um ensaio clínico aleatorizado, respeitando todas as etapas metodológicas do estudo, os resultados devem ser interpretados e generalizados com cautela.

\section{CONCLUSÃO}

Os resultados demonstraram que em um mês de treinamento com 10 repetições em força máxima de resistência mantida, aplicada em baixa velocidade, duas vezes/semana, foram suficientes para produzir ganho de força muscular nos grupos, verificado por aumento da força verificada em $\mathrm{mmHg}$ estatisticamente firmado. Houve melhora da força muscular e do desempenho funcional demonstrando pelo teste de sentar e levantar e melhora na qualidade de vida nos quatro itens avaliados através do SF-36. Houve correlação extremamente válida para a força em flexão com o teste de sentar e levantar, onde apesar de um ganho menor em detrimento a extensão estabeleceu-se correlação com o ganho funcional. Mais estudos com uma maior população e em graus angulares de isometria se tornam necessários para uma melhor compreensão da biodinâmica funcional nesta população.

Todos os autores declararam não haver qualquer potencial conflito de interesses referente a este artigo.

\section{REFERÊNCIAS}

1. Hall JE. Tratado de fisiologia médica. 12a. ed. Rio de Janeiro: Elsevier; 2011.

2. Soccol CP, Pinto LRC. Avaliação da força em idosos praticantes de musculação do Centro de convivência Padre Firmo. Rev Connection Line, no. 11:81-94. Disponível em: http://www.periodicos.univag.com.br/ index.php/CONNECTIONLINE/article/view/135 Acesso: em 30.set.2013.

3. Lustosa LP, Silva J, Coelho FM, Pereira DS, Parentoni AN, Pereira LSM. Efeito de um programa de resistência muscular na capacidade funcional e na força muscular dos extensores do joelho em idosas pré-frágeis da comunidade: ensaio clínico aleatorizado do tipo crossover. Rev Bras Fisioter. 2011;15(4):318-24.

4. Narici MV, Bordini N, Cerreteli P. Effect of aging on human adductor pollicis muscle function. J Appl Physiol. 1991;70(4):1277-81

5. Morgan D L, Allen D G. Early events in stretch-induced muscle damage Journal of Applied Physiology Published 1 December 1999.

\footnotetext{
6. Leite PF. Exercício, Envelhecimento e promoção de Saúde. Belo Horizonte: Health; 1996.

7. Powers SK, Howley ET. Fisiologia do exercício: teoria e aplicação ao condicionamento e ao desempenho. 3a. ed. São Paulo: Manole, 2000.

8. Meirelles MEA. Atividade física na terceira idade. 3a. ed. Rio de Janeiro: Sprint; 2000.

9. Souza LAC, Martins JC, Salmela LFT, Godoy MR, Aguiar LT, Faria CDC. Avaliação da força muscular pelo teste do esfigmomanômetro modificado: uma revisão da literatura. Fisioter Mov. 2013;26(2):437-52.

10. Omura S, Kerbauy RR, Caromano FA. Elaboração e aplicação de teste para avaliação do levantar e sentar em idosos saudáveis. Arq Ciênc Saúde Unipar. 2001;5(2):127-33.

11. Ciconelli RM, Ferraz MB, Santos W, Meinão I, Quaresma MR. Tradução para língua Portuguesa e validação do questionário genérico de avaliação de qualidade de vida SF-36 (Brasil SF-36). Rev Bras Reumatol. 1999;39(3):143-50.
} 